NASA Technicial Memonadum 105566

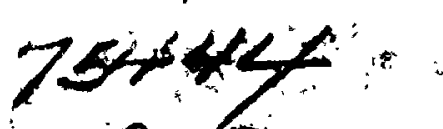
(

\title{
Predictive Onboard Flow Control for Packet Switching Satellites
}

Eric A. Bobinsky

Lewis Research Center

Cleveland, Ohio

Propined for the

14th International Communications Satellite Systems Conference sponsered by the American Institute of Aeronautics and Astronautics Washingtor, D.C., March 22, 1992

\section{NMSN}

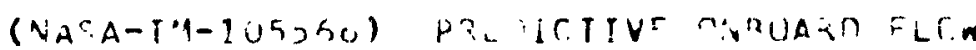
$\ln 2<-1037$ COIJTHL FOK PALKLT FWIICHING SATCLLITES 


\title{
PREDICTIVE ONBOARD FLOW CONTROL IN \\ PACKET SWITCHING SATELLITES
}

\author{
Eric A. Bobinsky ${ }^{1}$ \\ Space Electronics Division \\ NASA Lewis Research Center \\ Clevelond, Ohio 44135
}

\begin{abstract}
We outline two alternate approaches to predicting the onset of congestion in a packet switching satellite, and argue that predictive, rather than reactive, flow control is necessary for the efficient operation of such a system. The first method discussed is based on standard, statistical techniques which are used to periodically calculate a probability of near-term congestion based on arrival rate statistics. If this probability exceeds a preset threshold, the satellite would transmit a rate-reduction signal to all active ground stations. The second method discussed would utilize a neural network to periodically predict the occurrence of buffer overflow based on input data which would include, in addition to arrival rates, the distributions of pecket lengths, source addresses and destination addresses.
\end{abstract}

\section{Motivation}

Consider a node in a packet switched network characterized by $m$ inbound links and $n$ outbound links. Inbound packets are routed over a switching fabric to their appropriate outbound links based on their indiviciual destinatior addresses. We begin by making the general assumptions that packet lengths are variable and that we have no a priori information concerning the statıstical nature of the packet routing patterns.

Let $C_{i}$ represent the temporal capacity of link $i$. At any instant in time $t$, a link carries an amount of traffic equal to some fraction $\nu_{i}$ of its total capacity. In what follows, we define local buffer congestion to be the local conditing obtaining when the totality of inbound traffic destined for a particular outbound link exceeds the capacity of that link. More formally, local congestion occurs when

\footnotetext{
${ }^{1}$ Applied Mathematician, Communications Systems Branch Member, AIAA
}

$$
\sum_{i=1}^{m} v_{i}^{()} c_{i}>c_{j}
$$

where $v_{1}^{(0)}$ is the fraction of inbound traffic on link $i$ destinad for transmission on outbound link $j$.

The impact of local congestion on the performance of the network depends on its magnitude und on the length of time the condition persists. If we assume that the node contains some quantity of buffer memory for each outbound link, then the onset of congestion causes the buffer to fill to capacity, whereupon newly arriving packets are blocked. Thus, if blocking is used as a figure of merit, the length of time a node can sustain local congestion is proporiional to the amount of buffer memory it supports and the local input rate. Considerable research has been carried out on optimizing the amount of memory built into packetsuritching nodes given specific retwork operating protocols and performance specifications (cf. [1]).

Because of the stochastic nature of the inbound traffic, usually with respect to packet arrival rates, service rates, and destination routing requirements, overflows will occur with some finite probability. Two basic ayproaches to the problem are, 1), attempting to estimace the maximum duration of such events and provide surficient buffer to ninimize packet loss, and, 2), to use flow control techniques to minimize the probability of the occurrence of overflows. Typical network architectures incorporate various combinations of these (and other) methods to mitigate the effects of congestion. Many packet-oriented communications protocols require the retransmission of blocked packets, which can lead to the global condition of network congestion, wherein the average packet throughput delay becomes intolerably large.

It is difficult to efficiently adapt flow control schemes 
designed for low- to moderate-rate terrestrial networks to links characterized by high bandwidth-delay products, such as high-speed fiber optic links or geostationary satellite links. In particular, the advent of commercial communication sitellites capable of on-board packet routing poses serious challenges in efficiently processing packet iraffic routed through a satellite with the objective of minimizing both packet loss and throughput delay.

We consider the former to be the more siguificant problem. Common terrestrial protocols recognize the onset of congestion after the fact through detection of anomalously high throughput delay and/or traffic blocking. The volume of packets lcst before this congestion is brought under control, due to the relatively short length of terrestrial links, is quite small relative to that which would be lost on the much longer geostationary Earth-space link. Moreover, retransmission of lost packet- from ground stations to the satellite, again due to the inherently large propagation delay, can clearly have a severe impact on end-to-end performance (extremely severe in, for example, the case of go-back-n ARQ protocols [2]). On the other hand, the in situ queueing and transmission delay suffered by a packet as a consequence of onboard processing operations would be relatively inconsequential compared to the unavoidable propagation delay experienced by the packet. Thus, in this work, we consider the main problem to be that of preventing local congestion (cnboard buffer overflow) and subsequent packet blocking and loss by attempting to predict the onset of such a condition prior to its occurrence and controlling it through a rate-based, feedback control mexhanism.

It should be noted that we do not address the problem of admission control to the network, which is usually a function of the call setup procedire. In this work, we deal with those sessions which have already been admitted. We operate on the assumption that the true packet rate and routing distributions of an individual user requesting access are unknown at the time of call setup, thus the user traffic characteristics may change during the course of the session, possibly resulting in congestion.

We outline two approaches to controlling the rate of isbound (uplink) traffic destined for a particular outbound link (downlink) in a satellite system architecture supporting full, destination-directed packet routing and packet buffering. The first approach centers on statistical estimation and prediction, while the second is based on neural networks which capture finite time series behavior. Both approaches use time-history daty on the behavior of the arrival rate to predict the near-term occurrence of an overflow, at which time a rate-control (or throttle) signal is transmitted by the satellite to each ground station. We begin by developing a conceptual model of the system architecture.

\section{A System Model}

The general description of the packet switching node given above can be extended and formalized. Our goal is a simple architectural model which is "reasonable" in the sense that the number of (possibly unrealistic) assumptions needed conceming its operation is minimal.

As before, we assume that the architecture supports $m$ inbound and $n$ outbound links (the distinction is logical, not physical; that is, the satellite supports $m$ uplink channels and $n$ downlink channels). We make no assumption at this point concerning the satellite access method (i.e. TUMA, FDMA, etc.). The communications payload contains a switching fabric capable of routing individual packets from any uplink to any downlisk. Each downlink channel $i$ is preceded by an amount of store-and-forward buffer $b_{\text {i }}$ (which we will take as fixed, although we do not rule out dynamic allocation schemes). Placing the buffer memory between the routing switch and the downlink channel circuitry, though necessary to the specific formalism developed here, is not crucial to the basic approach.

A key requirement of the control mechanisms to be developed is that the system architecture support a common signalling channel or priority broadcast mode by which lowrate flow control commands can be transmitted from the satellite and received simultaneously by all active ground stations independently of user traffic.

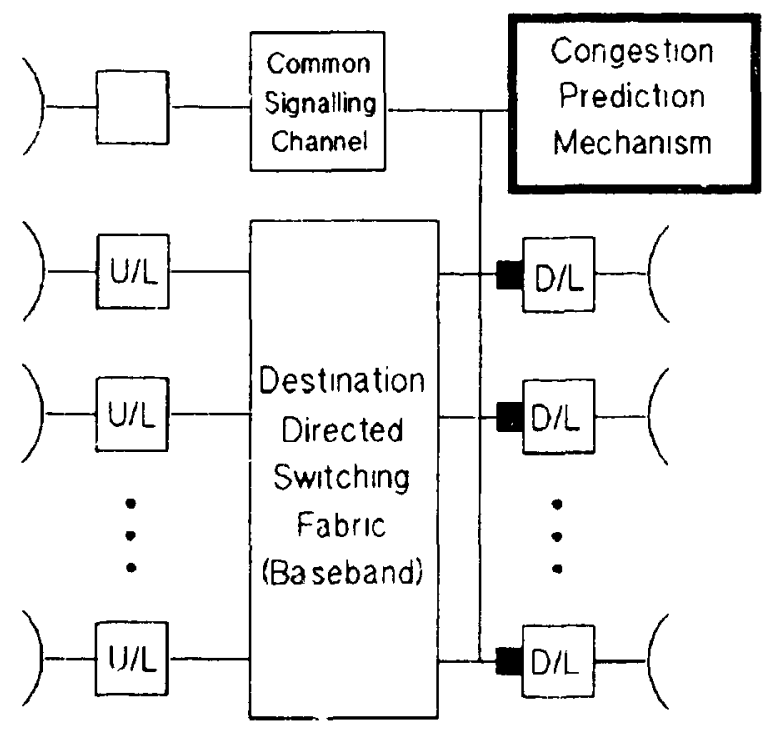

Fig. 1 
Figure 1 schematically illustrates the system model in the context of a geooric switching satellite architecture.

We next turn to a fundamental characteristic of any flow control model, which is the assumed nature of the behavior of the traffic arrival strenms. The traditional mathematical represiantation of packet arrivals, the Poisson promess model, requirs the assumptions that the interarrival tim ; between successive packets are exponentially distributed and that the key parameters (such as paclet length and destination) of successive packets are statistically independent. Slarily, these ascumptions are of questionable value in many (or ever - ost) realistic situntions; however, there are occasions in which they are usually valid. Kleiarock [3], for example, demonstratod that multiplexing a sufficiently large number of packet streams arriving from independent sources results in a composite stream exhibiting Markovian behavior.

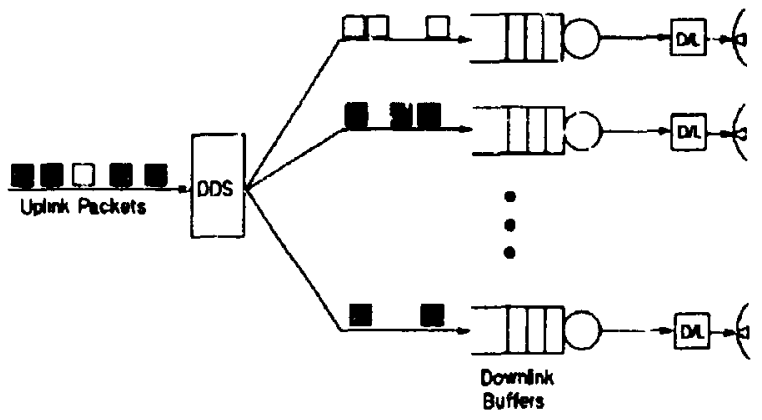

Fig. 2

The system model proposed can be viewed as a queveing system as illustrated in Figure 2. Here we represent the totality of uplink traffic as a single stream which is demultiplexed (in a sense) by the suitching fabric. In other words, we assume that the switch acts to randomly selext packets and route them to the downlink queues, thus resulting in $n$ individual arrival streams exhibiting Markovian behavior (in other words, packets arriving from independent sources are multiplexed by the switching fabric and appear at the output of switch in such a way that successive packets are no longer correlated). Depending on the actual architesture of the switching fabric, each downlink channel may well observe a packet stream which is (very nearly) governed by a Poisson distribution.

The importance of such behavior is most evident in the construction of steady-state queueing models of network nodes, particularly in the case of the $M / M / 1$ queve, in which both the packet arrivals and lengths are governed by a Poisson distribution with rate parameter $\lambda$. In the steadystate, the expected value of the number of puckets in the buffer is given by

$$
E(n)=\frac{\lambda / \mu}{(1-\lambda / \mu)}
$$

wheri $\lambda$ is the average arrival mate and $\mu$ is the average packet length. Although queveing models have been used vith much success to design communication networks, their obvious drawbak is the requirement that one can typically deal oxily with steady-xtate, average behavior. In attempting to develop a control mechanism to circumvent congestion, we are, in fact, almost exclusively concerned with the transient behavior of the system (aithough significant work has been done in the transient behavior of queves, most tractable aspects of the theory are restricted to steady-state analyses, cf. (44).

A some what better method of dealing with traffic streams which do not realily lend themselves to steady-state treatment is to assume that the arrivals are governed by nonstationary promability distributions. In the simplest case of the stationary Poisson model, the probability that $k$ packets will arrive within a time interval $\tau$ is given by the expression

$$
\operatorname{Pr}[K=k]=\frac{e^{-\lambda \tau}(\lambda \tau)^{k}}{k !}
$$

where $\lambda$ is the average packet arrival rate defined by

$$
\lambda=\lim _{t \rightarrow} \frac{\text { Exp. Arrivals in }[0, t]}{t}
$$

Pragmatically, $\lambda$ is assumed to exist and is generally taken to be a long-term average of arrival rates of an ergodic point process, and can vary significantly from shorter-terti or rapid fluctuations (characterised by higher-crder statistics). Longer-term fluchuations are characterized by more gradual changes in the value of the parameter of the governing probability distribution.

If, on the other hand, the arrival process is governed by

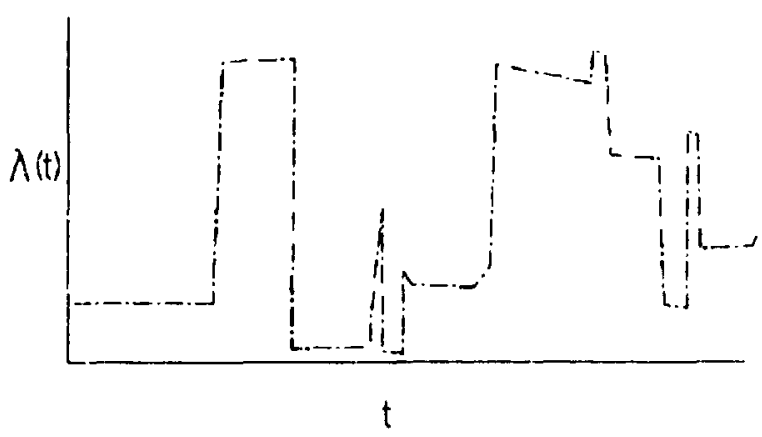

Fig. 3 
the nonstationary Poisson distribution (i.e. by the nonhomageneous Poisson process), the behavior is taken to result fiom stochastic time-dependency of the average rate parameter, viz: $\lambda=\lambda(t)$. Notice that $\lambda(t)$ may itself be a stochastic process of indeterminate statistics. Figure 3 illustrates a typical uncontrolled arrival stream subject to both types of behavior. Both short and long-term variations are clearly evident. Formally, the rate parameter $\lambda(t)$ is defined by

$$
\lambda(t)=\frac{d \Lambda(t)}{d t}
$$

whore $\Lambda(t)$ is termed the expectation function and is assumed 10 b: differentiable at an instant $t$. The probability of $k$ ar.ivals occurring in a time interval of length $\tau=[t, t+s]$ is now

$$
P r[K=k]=\frac{e^{-[\Lambda(t+s)-\Lambda(s)}[\Lambda(t+s)-\Lambda(t)]^{k}}{k !}
$$

where

$$
\Lambda(t+s)-\Lambda(t)=\int_{i}^{t+s} \lambda(u) d u
$$

or, by substitution,

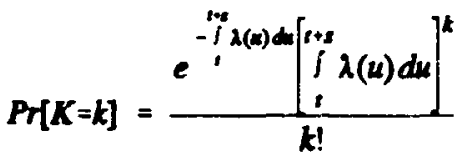

The motivation here is simply that we hope to realize bounds on the short-term transients (which are governed by higher-order statistics), whereas changes in the average rate parameter $\lambda(t)$, though still of a stochastic nature, will be driven by a deterministic, underlying structure. Learning and adapting to this structure is the essence of both the statistical and neural network feedback control mechanisms proposed. By developing a mecinanism which can learn and predict the relatively near-term behavior of $\lambda(t)$ and combining it with a state-variable representation of the average number of packets in queue at time $t$, we can attempt to predict the point at which congestion will occur and initiate rate-reduction in the arrival stream. This approach is only one of many filtering and prediction methods available through classical stochastic control theory; we choose it for its apparent operational simplicity. We elaborate on this approsch in Section III.

We digress to note ihat it is possibles to combune the time-varying arrival rate with the steady-state $M / M / 1$ queueing model to derive a state equation describing the tine evolution of the queue length. Filipiak [5] (among others) matches the stendy-state equilibrium point of the queueing model with that of the dynamic model to obtain the differential equation

$$
\dot{x}(t)=-\mu\left[\frac{x(t)}{1+x(t)}\right]+\lambda(t)
$$

which has the initial condition $x(0)=x_{0}$. This expression describes the evolution of the mean number in the queveing system over time, and does not, in general, account for short-term transirnts. However, if $\lambda(t)$ is taken as the ensemble average of the arrival process and it is known (or can be shown) that the variance of the process is relatively small , the state variabie can be a fairly accurate predictor of the dynamics of the system. If this is the crse, standard optimal control techniques (Hamilton-Jacobi) can be applied to the state evolution expression above to derive an optimal control to minimize the blocking probabilities [6]. We do not assume here that purely steady-state behavior will be sufficient to arcuratel'y predict the onset of congestion.

Our motivation for examining both statistical and neural network control mechanisms is that statistical methods are well-understood anc have been extensively analyzed and used. Thus, even though stochastic formulations must necessarily rely on certain working assumptions (i.e. be "model-based"), we are at least able to determine when such assumptions are-- or are not-- valid. On the other hand, neural networks are not yet well understood; a significant amount of trial and error testing is usually required to develop functional prototypes and the underlying theory is far from comprehensive. Nonetheless, the attraction of neural networks here lies in their potential for model-free estimation and prediction, coupled with their ability to distinguish patterns and trends which cannot be adeyuately captured by pr sbabilistic methods. We begin witb a proposed technique based on statistical estimation and prediction.

\section{An Approach to Statistical Predictive Control}

The basic statistical predictive flow control mechanism we propose is simply as follows: at time $t$, predict the probability of an overflow at time $t+s$. If the predicted probability metric exceeds a predefined threshold, transmit a throttle command to all active ground stations to effect an immediate reduction in the number of uplink packets destined for that downlink buffer.

We assume that the packet arrival rate is determined by counting circuitry at each output of the destination-directed switch (a sampling mechanism might also be used). It 


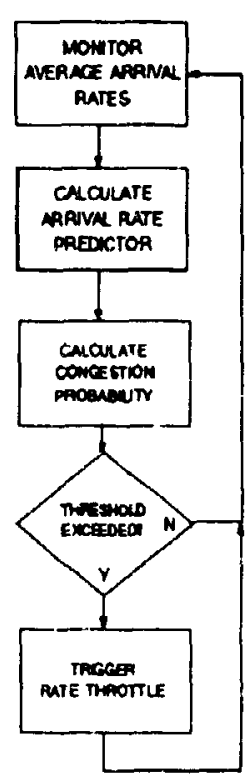

Fig. 4

appears that, due to the digital nature of the system, the measured value of the packet arrival rate should be relatively free of measurement noise, obviating the need for statistical filtering at that point. If this is not the case, then optimal filtering techniques (such as the well-known Kalman filter) must be used. The measured arrival rates represent the composite transmission rate of all active ground stations to each particular downlink buffer approximately $125 \mathrm{~ms}$ in the past. The task of our proposed prediction mechanism is to calculate the probability of congestion within the nearterm future (for a predefined window), compare that value with a preset threshold, and trigger the transmission of a throttle command if the threshold is exceeded. Figure 4 illustrates the algorithmic flow.

Our definition of congestion probability is based on the nonhomogeneous Poision process model described sbove. As before, let $b_{i}$ be the total amount of buffer available at the downlink queue and let the amount of buffer in use at time $t$ be $N_{1}$. The probability of congestion can be definea as the probability that more traffic will arrive at the queve in the interval $[t, t+8]$ than can be accommodated, which is expressed by

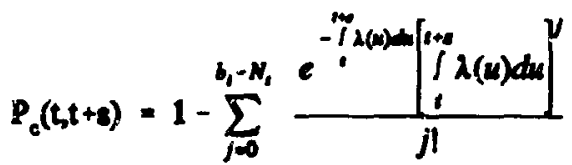

Note that, for simplicity, we take the conservative 4pprach of neglecting separtures from the buffer during the intervai. Numerical computation of $P_{\alpha}(t, t+8)$ is relatively straigntforward. The problera, then, is in cotimating and predicting the value of $\lambda(t)$ for the near-term window.

The shoice of an appropriste prodiction scheme is, unfortunately, rather highly dependent on the dynamics of the arriving traffic stream. If the traffic pattern seen at the downlink queue is relatively bomogeneous on, say, successive days (as is typically the case of interactive computer use, for example), then it may be convenient to average the arrival rates over several days and derive a smooth, polynomial approximation $\lambda^{*}(t)$ of $\lambda(t)$ using a technique such as maximum likelibood estimation.

On the other hand, if the arrival process is characterized by large variations over the mean, then basing the estimate on average values would be insufficiently accurate for purposes of control. Alternate estimation procedures (of which there are many) must be chosen to take advantage of any determinusic structure underlying the arrival process. Possible choices include the Kalman filter, the Sage-Husa filter, LMS adaptive filters, and local linear predictive techniques (cf. [7], [8]).

For example, a simple linear predictor expresses the value of $\lambda^{*}(t)$ by linear extrapolation through two previous data points. Thus,

$$
\lambda^{*}(t)=\left(t-\tau_{1}\right)\left(\frac{\hat{\lambda}\left(\tau_{2}\right)-\hat{\lambda}\left(\tau_{2}\right)}{\tau_{2}-\tau_{1}}\right)+\hat{\lambda}\left(\tau_{1}\right)
$$

where $\hat{\lambda}(t)$ is the measured value of $\lambda(t)$ at $\tau_{i}$. A little elementary calculus gives us that

$$
\begin{aligned}
& \int_{1}^{s+s} \lambda^{*}(u) d u \\
& =\frac{\hat{\lambda}\left(\tau_{2}\right)-\hat{\lambda}\left(\tau_{1}\right)}{\tau_{2}-\tau_{1}}\left(\frac{s^{2}+2 i s}{2}-s \tau_{1}\right)+s \hat{\lambda}\left(\tau_{1}\right)
\end{aligned}
$$

In this case, expression (10) can be computed quite simply either directly or using series approximations.

Although in certain well-behaved cases a linear predictor can perform quite well, typically higher-order prodictors are necessery. In addition, it is probable that the higher-osder statistics of the arrival stream may have to be taken into account by the prediction mechanism, which raises several difficulties in applying a classical probabilistic approach. The overall objective, of course, is to maximize the 
efficiency of the prediction mechanism; that is, to determine $P_{c}(t, t+8)$ such that it exceeds its threshold value largely when the actual arrival rate will result in an overflow, and rarely otherwise.

Determination of an approximation function $\lambda^{*}(t)$ of $\lambda(t)$, though beyond the scope of this paper, is one of the key factors in the success of the proposed control mechanism. An important consideration here is the accuracy of the value of $\lambda^{*}(\mathfrak{i})$ within the near-term window-- in other words, bow well does the estimation scheme capture the structure of short-term fluctuations? Ideally, we wish to minimize the probability of congestion due to these short term trausients. One possible (and purely spectlative) approach to this problem is to view the short-term transients as yet another stochastic process, governed by its own set of statistics. Thus one can envision to separate prediction mechanisms, one geared to prodicting long-term behavior, the other to predicting short-term fluctuations.

Several aspects of this scheme will require resolution. First, the time which must elapse between the transmission of a throttie command ard its receipt by a ground station is approximately 125 milliseconds (during which time 125 milliseconds worth of truffic is uplinked to the satellite), placing a bound on the minimum prediction window.

Second, we assume that the data avaiiable to the prediction mechanism will consist of a finite amount of time series data concening the arrival rate, distribution of packet lengths, sources and destinations.

Third, the action carried out by a ground station upon receipi of a throttle command is taken to be a (possibly total) reduction in the transmission of packets destined for the affected queue (whereupon such packets are buffered at the ground station, resulting in backpressure along the terrestrial links to the attached nodes). We stould note here also that a possible consequence of this backpressure is that upon release of the traffic, the hypothetical Poisson model may become a batch arrival process and must be treated differently.

Fourth, the length of time rate reduction is executed at the ground stations must be resolved. Fifth, and finally, the optimal value of the presef probability threshold must be determined on an application-specific basis by trading off link efficiency and overall quelity of service requirements.

\section{Neural Network Predictive Control}

Time-serics prediction using neural networks is a relatively new and active research area. Notable work in the prodiction of highly nonlinear (and chaotic) time-senes has been performed by Jones, et ai. [9], among others.

A 1988 report by Elman [10] describes a simple, partially recurrent neural network architecture designed to predict the successive elemenis in a sequence (i.e which prodicts the state of the sequence at time $t+1$ given the current state and the state at time $t-1$ ). The key feature of this architecture is the addition of a hidden layer of neurons (termed the context layer) which provides the network with memory. Although developed for application to language prucessing, the ability of the network to make predictions based on finite time series appears to constitute an appropriate basis for the control of packet arrival streams. Figure 5 schematically illustrates Elman's neural network architecture.

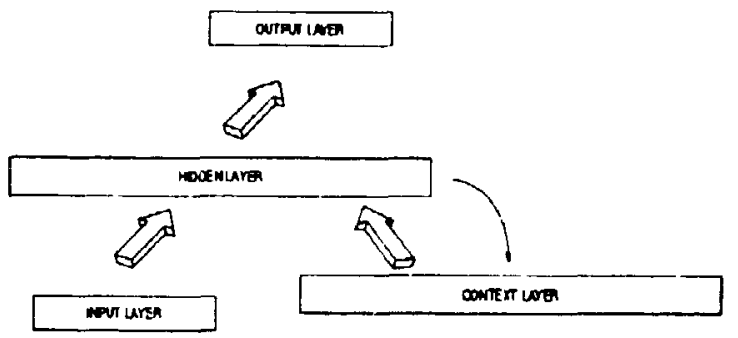

Fig. 5

The operation of the network is fairly straightforward. Ignoring the context layer for the moment, the input, hidden, and output layers would function as a simple, feedforward neural net. B, copying the contents of the hidden layer back onto the cuntext layer at each time step (say, at time $t$ ), the network stores the state of the hidden layer at time $t$. During the $t+1$ st processing step, the context information is propagated back into the hidden layer, essentially "influencing" the current state with time-history data from time $t$. If the network is being trained (Elmen utilizes the method of hackpropagation), then the forward connection weights are adjusted in the standard manner. The recurrent connections are fixed at unity and are not affected by the training.

Because the context layer is continually updated by copies of the current hidden layer, a time-history is maintained in the network. In particular, even though only a single context layer is used, historical events that are of large magnitude (in some sense) can continue to influence the hidden layer over more than a single time step.

Thus, returning to the problem at hand, the neural net would serve as a predictor of overflows. A singular advantage to this approach over the statistical method is the ease of using not only arrival rates as a basis for predict:on, 
but also such information-rich data as the distributions of packet lengths, source addresses, and destination addresses. In addition, this approach does not require the assumption of a Markovian arrival stream. Indeed, positive correlations between successive fackets is in itself a sourcs of data which could be used by an intelligent prediction mechanism; in fact, Elman's work is sequence analysis seeks just such correlative behavior.

Operationally, we envision that the neural network would be trained with input vectors consisting of the arrival statistics given above and a binary output vector indicating whether or not congestion occurred within the specified prediction window. 'Typically, two noural networks would operate in tandem, with one undergoing online training while the other generates a binary signal corresponding to its prediction of either an overflow condition or nominal operation. Output of a value indicating a predicted overflow conciition would, as in the statistical prediction mechanism, result in transmission of a throttle signal to the active ground stations.

A ciear advantage to using neural networks for this approach is that one need not be concerned about formally discriminating between first and second order arrival statistics, and by the difficult problem of combining several distributions into a multivariate prediction model. Thus, it may be possible to devise a much more efficient predictor using the neural network's inherent capability to train on multivariate input data.

Neural networks are by no means magical, however, and careful consideration nust be given to the appropriate implementation of such a network for the specific application. As this paper is preliminary, we do not yet have a firm grasp of the precise network architecture that is required to predict congestion. It does appear, however, that Elman's architecture, through its ability to efficiently use time-history data, offers significant promise in the ares of recursive estimation.

\section{Summary}

We outlined two alternate approaches to the predictive control of traffic arrival to a packet switching satellite. Wa believe such an approach to controlling congestion is necessary for the operational efficiency of such systems, since allowing the loss of earth-to-space pucket traffic wculd result in serious performance degradation, perhaps crusing the system performance to rall below the required quality of service.

The first approach was based on standard statistical methods for dealing with traffic flows in computer and cummunication networks. A nonstationary distributiou was used to derive a simple expression for the probability of a local overflow which could, in theory, be either periodically or continuously calculated by an onboard processor and compared to preset (and fossibly dynmic) thresholds to initiate transmission of rate control signals to the ground station population.

The second method suggests using a novel class of neural network developed by Elman to predict an overflow based on a number of available traffic arrival statistics, including the arrival rate and distributions of packet lengths, sources, and destinations. Thi proposed neural network architecture utilizes a recurrent context layer of neurons to capture recent past behavior of the arrival stream and incorporates this into its ifeedforward) predictive output.

Efforts are underway by the author to test the effectiveness of both methods through the tool of computerbased simulation. Of interest are not only the practicality of the methods, but also their robustness to dynamic changes in the intensity, time-variations, and statistics of the traffic flow. Preliminary results using the simple linear predictive non-homogeneous Poisson process for random arrivals based on quasi-deterministic structures (simple periodic functions with a uniformly-distributed random component) appear promising. Evaluation of the control mechanisms in 2 more realistic networking scenario has recently begun. The problem of an effective estimator for $\lambda^{*}(t)$ bas not yet been addressed.

Potential applications for this work include several projects underway at NASA Lewis Research Center (such as the Information Switching Processor and Autonomous Network Controller) as well as applications to envisioned future satellite services supporting packet and fast-packet routing, such as ISDN and B-ISDN.

\section{Acknowledgements}

The author is indebted to Dr. Stanley C. Ahalt of the Ohio State University for introducing him to Elman's recurrent neural network architecture.

\section{References}

[1]. Li, S.: Overload Control in a Finite Message Storage Buffer, IEEE Trans. on Commun., 37:1330-1338, Dec., 1989.

[2]. Bertsekas, D. and R. Gallager: Dala Networks, Prentice-Hall, Englewood Cliffs, NJ. 1987.

[3]. Kleinrock, L.: Queuing Systems Volume I: Theory, Wiley, New York, NY. 1976. 
[4]. Wolff, R.: Stochasric Modeling and the Theory of Queues, Prentice-Hall, Englewood Cliffs, NJ. 1989.

[5]. Filipiak, J.: Moaelling and Control of Dynamic Flows in Communication Networks, SpringerVerlag, New York, NY. 1988.

[6]. Tipper, D. and M. Sundareshaj: Numerical Methods for Modeling Computer Networks Under Nonstatıonary Conditions, IEEE J. Sel. Areas Commun., 8:1682-1695, Dec., 1990.

[7]. Chemouil, P. and J. Filipiak: Kalman Filtering of Traffic Fluctiations for Real-Time Network Management, Proc. IFAC Large Scale Systems: Theory and Applications,827-832, Zurich, 1986.

[8]. Farmer, J.D. and J. Sidurowich: Predicting Chantic Time Series, Phys. Rev. Lett., 59:845-848, 24 Aug., 1987.

[?]. Jones, R.D., et al.: Function Approximution and Time Series Prediction with Neural Networks, LAUR 90-21, Los Alamos Nall. Laboratory, New Mexico, 1990.

[10]. Elman, J.: Finding Structure in Time, CRL Tech. Rep. 8R01, University of California, San Diego. April, 1988. 


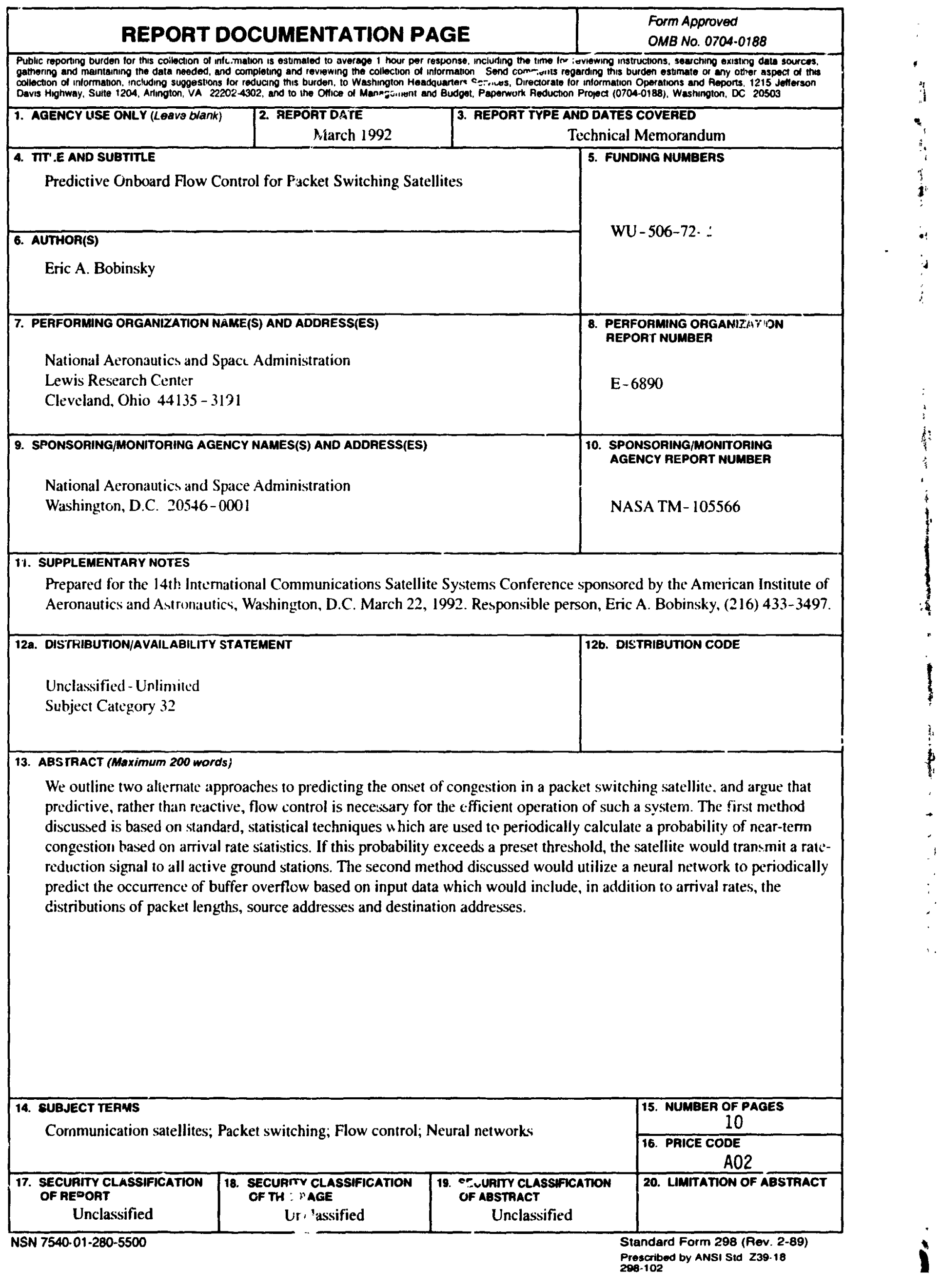

